\title{
Robust Simulation and Nonlinear Performance
}

\author{
Michael Kantner John Doyle \\ Department of Electrical Engineering \\ California-Institute of Technology \\ Pasadena, CA 91125 \\ kantner@hot.caltech.edu \\ doyle@hot.caltech.edu
}

\begin{abstract}
Robust simulation, defined as the simulation of sets, allows the computation of a system's global properties. By simulating entire sets, instead of individual points, performance guarantees can made. While exact algorithms for robust simulation are computationally prohibitive, reasonable approximations which preserve performance guarantees exist.

An approximate solution, which provides an upper bound on performance, is tested on a large number of systems. In general, the upper bound is close to the best lower bound computed by search. Furthermore, when the bounds differ, several techniques exist to improve the upper bound.
\end{abstract}

\section{Introduction}

Historically, simulation has played a large role in nonlinear system analysis. Stability and performance are often studied by simulating a large number of initial conditions and noise signals. However, the results of these simulations do not guarantee stability or performance, since the behavior for other initial conditions and noise signals is not examined.

Robust simulation addresses this limitation. Instead of simulating a single initial condition and noise signal, a set initial conditions and noise signals are simulated at once. If the robust simulation meets the performance requirement, then the system meets the performance requirement.

As with any general nonlinear problem, some assumptions are required to ensure reasonable computational cost. For robust simulation, finite time horizon problems for discrete time piecewise linear systems are considered. All simulation based techniques require finite time horizons and the discrete time piecewise linear restriction is less onerous than it sounds.

Piecewise linear (PL) systems are interesting for a variety of reasons. They are a conceptually simple extension of linear systems and are easy to implement and simulate. At the same time, PL systems can be used to approximate general nonlinear systems and can exhibit complex behavior.

PL system theory has been studied for over a decade. In 1981, Sontag suggested the use of PL systems for nonlinear regulation [4] and developed a PL algebra.[5] Recently, Pettit and others have studied continuous time PL systems. [3] Unfortunately, there has been little progress developing computational tools for PL system analysis. In fact, Sontag demonstrated that computing practically all interesting PL system properties is NP-hard.[6]

\section{Robust Simulation}

Traditional simulation maps a single point in initial condition and noise space into a single final condition. We define robust simulation as the mapping of all initial conditions and noise signals into a set of final conditions. By calculating all possible trajectories, one can make guarantees about the system's global behavior. Traditional simulation only gives local information. Essentially, robust simulation maps sets to sets, while traditional simulation maps points to points.

0-7803-3590-2/96 \$5.00 @ 1996 IEEE
The mapping of sets introduces a new problem, set representation, into the simulation process. For linear systems, this is not a difficult issue, since polyhedra map to polyhedra via matrix multiplication. However, nonlinear systems can map polyhedra into anything. For efficient computation, simple set descriptions and mapping rules are required.

The set representation issue is simplified by requiring discrete time piecewise linear systems, which map polyhedra to polyhedra via simple matrix operations. This simplification also eliminates two problems encountered in traditional simulation: step size determination and derivative approximation. This allows us to focus on the issues central to robust simulation, set representation and computational complexity.

\section{Piecewise Linear Systems}

A piecewise linear (PL) system is defined over some subset, $Z$, of a finite dimensional real vector space, $\mathcal{R}^{n} . Z$ is the union of a finite number, $l$, of closed polyhedra, denoted $R_{i}$. Each $R_{i}$ is defined by a finite number of linear inequalities, $f(x) \geq a$. In each $R_{i}$, an affine state transition map is defined by

$$
x(k+1)=A_{i} x(k)+B_{i}+N_{i} w[k], \quad x \in R_{i}, \quad i \in 1 \ldots l \text {. (1) }
$$

The noise signal, $w[k]$, is chosen to be in $l_{\infty}$ to simplify computations. The methods in this paper trivially extend to timevarying $P L$ systems, as well.

This definition simplifies computer implementation, but leads to a minor technical problem. Since the polyhedra are closed and may share boundaries, the mapping is not always well defined on the boundaries. While it is possible to create a PL system that exploits the behavior on the boundaries, this is generally not the case. Because this technical issue is easily resolved (see [4]), and only clutters the description of the problem, it will be ignored for the remainder of this paper.

By construction, each $R_{i}$ is convex and bounded by hyperplanes. While the number of hyperplanes, $c$, bounding each $R_{i}$ may vary, $c$ must be greater than $n+1$ (to define a simplex) and generally $c=2 n$ (to define a hyperrectangle). The notation $\mathcal{S}_{j}$ denotes a finite set of polyhedra in $\mathcal{R}^{n}$ at time $j$.

\section{Robust Simulation Algorithm}

The robust simulation algorithm answers the following:

For a given set of initial conditions, denoted $\mathcal{S}_{0}$, what are all possible final conditions, denoted $\mathcal{S}_{f}$ ?

By assigning a norm on $\mathcal{S}_{f}$, this becomes a performance measure.

The direct approach to calculating $\mathcal{S}_{f}$ has exponential growth in the computation as a function of $t$. To see this, start with $\mathcal{S}_{0}$, map forward one time step, and call the new set $\mathcal{S}_{1}$. Assuming (for notational simplicity) that $\mathcal{S}_{0}$ contains at most $l$ convex regions, there are at most $l^{2}$ convex sets in the $\mathcal{S}_{0} \cap R_{i}$. Since no restrictions are placed on (1), each of these convex sets can then map into all of the $R_{i}$. Thus, $\mathcal{S}_{1}$ can contain up to $l^{2}$ convex sets and $\mathcal{S}_{1} \cap R_{i}$ may contain $l^{3}$ sets. At any time step $j, \mathcal{S}_{j}$ can contain as many as $l^{j+1}$ independent convex sets. Repeating this process to form $\mathcal{S}_{t}$ yields, 
possibly, $l^{t+1}$ sets. All of these mappings can be computed by simple matrix operations and linear programming.

By slightly modifying the direct approach, a polynomial time bound is obtained. The fundamental idea is to limit the number of regions in $\mathcal{S}_{j}$ at every time step by restricting $\mathcal{S}_{j}$ to have a fixed number of convex sets in each $R_{i}$. The restrictions placed on $\mathcal{S}_{j}$ determine the tightness of the bound. The result contains $\mathcal{S}_{f}$, though the approximation may be conservative.

Given $\mathcal{S}_{j}$, the first step is to form a manageable approximation $\mathcal{T}_{j} \supseteq \mathcal{S}_{j} . \mathcal{S}_{j+1}$ is then calculated from $\mathcal{T}_{j}$. By restricting the number of sets in the approximation, exponential growth is avoided. By definition, $\mathcal{T}_{j}$ contains $l^{\gamma+1}$ convex sets, and there are $l^{\gamma}$ convex sets in $\mathcal{T}_{j} \cap R_{i}$. The meaning of $\gamma$ will be described later. Though the details are omitted due to space constraints, $\mathcal{T}_{j}$ can be calculated by linear programming.[1]

\section{Algorithm Refinements}

The accuracy of the approximation is directly related to the amount of extra volume added when forming $\mathcal{T}_{j}$. Two factors affect this: the number of convex sets in each $R_{i}$, and the number of hyperplanes, $c$, used to bound each region. By increasing each of these, accuracy may be improved.

As defined earlier, $\mathcal{T}_{j} \cap R_{i}$ contains $l^{\gamma}$ convex sets. The value $\gamma$ can be considered a history parameter. For $\gamma=0$, one does not consider what regions a set mapped from before arriving in the current $R_{i}$. For $\gamma=1$, one looks at where the set was one time step prior to the current time step. In this case, each $\mathcal{T}_{j} \cap R_{i}$ contains $l$ convex sets, each approximating the sets that came from a specified $R$. $\gamma$ determines how many previous time steps play a role in forming the approximation $\mathcal{T}_{j}$. As $\gamma$ approaches $t$, the approximation approaches the exact solution. When $\gamma=t$, the exact solution is obtained.

An equally important variable is the number of hyperplanes defining a convex set, $c$. Assuming hyperrectangles, the basic algorithms required $2 n$ bounding surfaces. In general, this is $2 n(\gamma+1)$. The bounding hyperplanes must contain those of the current region, $R_{i}$, and the bounds of the previous regions considered after the mapping law is applied. This way, a hyperrectangle can be exactly covered after mapping $\gamma$ time steps.

Since $c \geq 2 n(\gamma+1)$, an ad hoc approach for improving the approximation is to add more bounding surfaces according to some heuristic. In general, additional hyperplanes should differ greatly from existing hyperplanes.

Additionally, other generic bound improvement techniques, such as branch and bound are applicable.

\section{Computational Complexity}

The robust simulation approximation requires solving $t \mathrm{cl}^{\gamma+2}$ linear programs. $\mathcal{O}\left(n^{4}\right)$ public domain linear program solvers are available and $\mathcal{O}\left(n^{3.5}\right)$ algorithms have been proposed.[2] With $c=2 n$, the default when using hyperrectangles, $\gamma=0$, and an $\mathcal{O}\left(n^{4}\right)$ linear program solver, the overall complexity is $\mathcal{O}\left(t l^{2} n^{5}\right)$.

Memory usage is $\mathcal{O}\left(l^{\gamma+2} n c\right)$. At any time, up to $l^{\gamma+2}$ linear programs must be stored in memory. These linear programs require memory proportional to nc. Since the results from previous time steps do need to be saved, memory usage is independent of $t$.

In general, performance is much better than $\mathcal{O}\left(t l^{2} n^{5}\right)$. This worst case performance assumes that each region $R_{i}$ maps into every other region at each time step. Many systems map only into adjacent regions or themselves at each time step. By calculating what regions flow into other regions in advance, the number of linear programs solved at each step can be greatly reduced.

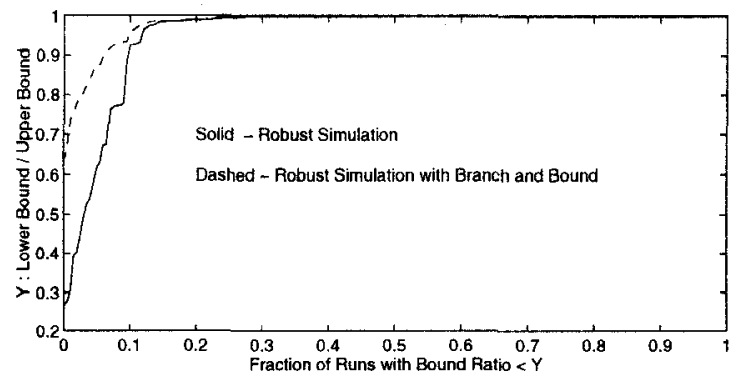

Figure 1: Nonlinear performance bound ratios

\section{Examples}

To evaluate the algorithm, tests were run on a set of randomly generated systems. The random systems were discretizations of 5 th order continuous systems with one saturation nonlinearity and no noise. Systems were simulated for 30 time steps. The performance measure chosen was $|x[30]|_{\infty}$. The initial condition set was $|x[0]|_{\infty} \leq 1.3$. This is a reasonable set of test problems that are moderately challenging for the algorithm. This is neither the hardest nor the easiest class of problems known.

Any traditional simulation gives a lower bound on the worst case performance. To achieve a large lower bound, gradient search was combined with random simulation. The upper bound was calculated by robust simulation with $\gamma=0$. If the bounds differed by more than $10 \%$, naive branch and bound was applied until the bounds differed by less than $10 \%$ or 50 branch steps were taken.

The measure of algorithm performance, shown in Figure 1, is the ratio of the lower bound to the upper bound. Ideally, the ratio is always 1 . In general, ratios greater than 0.9 are acceptable. For $89 \%$ of the runs, no branching was needed to obtain acceptable results. For the $11 \%$ of the runs where branching was needed, branch and bound greatly improved the bound ratio. These results can be greatly improved by better selection of branch cuts.

\section{Conclusions}

This work has demonstrated that robust simulation is a powerful tool for nonlinear system analysis. By calculating all possible trajectories for a set of points, measures of the system's global performance are obtained. The the exact solution has exponential computational cost, reasonable approximations can be computed in polynomial time. These approximations are generally very good, and can be refined by a variety of methods if needed.

\section{References}

[1] M. Kantner. Robust simulation home page. Available online at http: \hot.caltech.edu\ kantner \robustsim.

[2] N. K. Karmarkar. A new polynomial-time algorithm for linear programming. Combinatorica, 4:373-395, 1984.

[3] N. Pettit and P. Wellstead. A graphical analysis method for piecewise linear systems. In Proc. IEEE Conference on Decision and Control, pages 1122-1127, December 1994.

[4] E. Sontag. Nonlinear regulation: The piecewise linear approach. IEEE Transactions on Automatic Control, 26(2):346-357, April 1981.

[5] E. Sontag. Remarks on a picewise-linear algebra. Pacific Journal of Mathematics, 98(1):183-201, 1982.

[6] E. Sontag. From linear to nonlinear: Some complexity comparisons. In Proc. IEEE Conference on Decision and Control, pages 2916-2920, December 1995. 